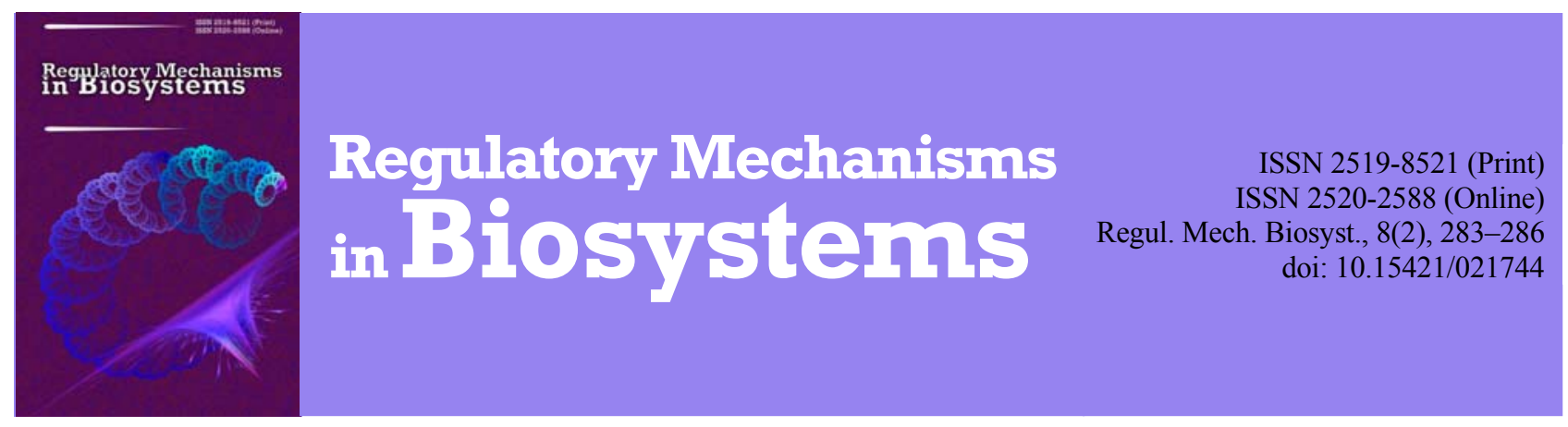

\title{
The influence of radio-mimetic chemical mutagen on the chromosomal complex of winter wheat cells
}

\author{
M. M. Nazarenko \\ Dnipro State Agrarian and Economic University, Dnipro, Ukraine
}

Article info

Received 12.03.2017

Received in revised form 28.04.2017

Accepted 07.05.2017

Dnipro State Agrarian and Economic University, Sergiн Efremov Str, 25, Dipro, 49600, Ukraine. Tel.: +7-095-848-53-86

E-mail:nik_nazarenko@ukr.net

\begin{abstract}
Nazarenko, M. M. (2017). The influence of radio-mimetic chemical mutagen on the chromosomal complex of winter wheat cells. Regulatory Mechanisms in Biosystems, 8(2), 283-286. doi:10.15421/021744
\end{abstract}

In this article we report the results of our investigation of cytogenetic parameters of changes in the chromosomal complex of new Ukrainian winter wheat varieties and some relationships between values of cytological indexes and different concentrations of DAB (1,4-bis-diazoacetyl butane). Analysis of chromosomal aberrations following mutagenic action of any kind of mutagen by the anaphase method is one of the most widely investigated and most precise methods which can be used to determine the fact of mutagenic action on plants and identify the nature of the mutagen. We combined in our investigation sensitivity of genotype to mutagen using cytological analysis of mutagen treated wheat populations with the corresponding different varieties by breeding methods to reveal their connections and differences, specific sensitivity to mutagenic action on the cell level. Dry seeds of 7 varieties and 1 line of winter wheat were subjected to $\mathrm{DAB}$ in $0.1 \%$ and $0.2 \%$ concentration, which is standard practice for mutation breeding of winter wheat. We investigated rates and spectra of chromosomal aberrations in the cells of the primary root tips of winter wheat during mitosis. The coefficient of correlations between the rate of chromosomal aberrations and the concentration of $\mathrm{DAB}$ was at the level $0.6 \%$. Fragments/bridges ratio is a clear and sufficient index for determining the nature of the mutagen agent. We distinguished the following types of chromosomal rearrangements: chromatid and chromosome bridges, single and double fragments, micronuclei, and lagging chromosomes. Investigation of DAB action confirmed the reliability of the fragments-bridges ratio (prevalence of fragments over bridges for chemical mutagens and vice versa for gamma-rays) for identification of the nature of the mutagen. Complicated (or combined) aberrations, micronucleus, lagging chromosomes were not observed for some varieties under DAB action. Genotypes selected after action of chemical mutagens are less sensitive to recurrent mutagenesis with chemical mutagens.

Keywords: chromosomal aberrations; recurrent mutagenesis; wheat; genotype

\section{Introduction}

DAB (1,4-bisdiazoacetyl butane) as a mutagen is not so widely used in breeding practice as the mutagens previously investigated in our experiments (gamma-rays and nitrosoalkylureas), but it more suitable for use in the reverse genetic approach in modern functional genomics and for programmes for genetic improvement of indigenous cultivars in South-East Asia (FAO-IAEA programmes for coordination of research into chemical mutagenesis) (Shu et al., 2011). DAB as a mutagen factor is traditionally related to radio-mimetic chemical mutagens due to the similarity of appearance of mutagenic action of this mutagen and physical mutagens such as gamma- and x-rays (Eiges, 2013).

Mutagenic effects on the level of the cell and the plant as a whole are the key factors which limit both winter wheat productivity for agricultural purposes and the number of families for the breeding programme to obtain next generation material for identification and selection of mutants. The consequences of mutagen action on the cell level (chromosomal aberrations) are closely connected with the future mutation rate. The influence of the action of mutagens depends on the following parameters: physiological parameter of the object of mutagenic action, genotype of the object, type of mutagenic action (acute, chronic), nature of the mutagen, doses or concentration of the mutagen, fractional of dose or concentration, time of exposure, concentration or presence of free active oxygen, temperature and other environmental conditions (Zhang et al., 2015; Nazarenko, 2016).
This article is the third part of our investigation of recurrent mutagen treatment of winter wheat varieties. In the previous parts we researched the effects of genotype-mutagen interaction following gamma-ray irradiation and nitrosomethylurea (NMU) nitrosoethylurea (NEU) (Nazarenko, 2016; Nazarenko, Izhboldin, 2017).

Recurrent mutagenesis includes the exposure to mutagenic action of the progeny of plants that have been treated by mutagens in previous generations. The strategy of treating the progeny of previously treated plants is well-known as recurrent action. Based on the assumption that recurrent action would increase both the rates and spectra of mutation events, recurrent mutagen treatment was the subject of widespread investigation from the 1940s through to the $1960 \mathrm{~s}$, a period of almost thirty years. It was expected that recurrent treatments would further broaden the induced genetic variability exploitable in crop improvement and increase the chances of obtaining some types of valuable mutations. Researchers studied a wide range of mutagens including different types of physical mutagens (different types of radiation) and the chemical mutagen (EMS); the alternation of EMS with irradiation was also studied. The results of these experiments did not bear out the expected results and were at best mixed. In most cases, radiosensitivity, mutation rate and spectra remained unaffected by repeated irradiation of subsequent generations (Ali Sakin et al., 2005; Waugha, 2006). In our research we used other types of chemical mutation factors and alternation these mutagens with gamma-rays. And we obtained other valuable results related to reduction in radiosensetivity and 
mutagen depression but also decrease in mutation rate in case of repeated exposure to the same mutagen. In the case of mutagen alternation we found a usual, normal reaction to mutagenic action (Nazarenko, 2016; Nazarenko and Izhboldin, 2017).

In modern scientific practice three main methods are exploited for investigation of mutagenic action on the chromosomal level. The first, the oldest one, is the observation of different types of chromosomal aberration which appear in mitotic cells during cell division. The second, FISH (fluorescence in situ hybridisation), is based on hybridisation of chromosome by specific DNA probes to metaphase preparations followed by detection with immuno-staining procedures. This technique allows detection of translocations involving different painted chromosomes (Jovtcheva et al., 2002). The third one is the micronucleus (MN) test in detecting small micronuclei compared with untreated and treated objects (Juchimiuk-Kwasniewska et al., 2011).

In our research we used the first method because of the advantages of this approach, which are simplicity, objectivity of the results, its reliability and its ability to assess the wide variety of different mutagens (Karthika and Subba, 2006; Albokari, 2014).

Analysis of the variability of chromosomal aberrations following mutagenic action of any type of mutagen by the anaphase method is one of the best-known and the most precise methods which can be exploited for determining the fact of mutagenic action and identifying the nature of the mutagen (Lifang et al., 2001; Adlera et al., 2004; Ukai, 2006). For example, this method is widely used for determining a radionuclide's pollution of the environment, its level, the danger of this pollution, as well as for identification of the optimal doses of radiation and concentrations of chemical mutagens in breeding practice (Ahloowalia et al., 2004; Nazarenko, 2017). The relation between clastogenic adaptation shown in reduction of chromatid type of aberrations, micronuclei and changes in number of chromosomes in cells is the manifestation of the clastogenic effect (Grant and Owens, 2001; Bignold, 2005).

Influence of different types of chemical mutagens or any type ionizing radiation according to this method can be analyzed by the number and kinds of chromosomal damage (Rakhmatullina, 2007). Both structural and numerical aberrations occur spontaneously due to intraneous and extraneous factors and appear during the cell division of the mitotic cycle (Nikolova et al., 2015).

Chromosomal rearrangements leading to visible mutations or modifications were first described in plants by de Vries (de Vries, 1918). A break in either single (resting stage) or divided (prophase) chromosomes is the consequence of direct action on the chromosome by the ionization produced by any elementary particle. Such a break may remain and give rise to terminal deletion, rejoin in the original position (restitution) or join with an indigenous break in the same or different chromosome to produce various types of rearrangements (Natarajan, 2005).

Changes in chromosome number and structure in mitotic cells are initiated after irradiation. Chromosomal changes result from damage, through exchanges, laggards and anaphase bridges, dicentric and centric ring formations, terminal fragments with telomeric signal at only one end and interstitial fragments that appear as double minutes without any telomeric signals changes in irradiated mitotic cells (Bolzarn and Bianchi, 2006; Rakhmatullina, 2007).

Together with genetic mutations, chromosomal aberrations may be generated in the form of rebuilding of different structures (deletions, inversions, translocations, etc.) (Shu et al., 2011). Translocation, a type of aberration which possibly results from the rejoining of broken chromosome parts, is the most important for mutation breeding on the chromosome level. Cytological aberrations observed in cells mitosis include the production of micronuclei and other types of chromosomal changes (Ukai, 2006; Shu et al., 2011).

All the effects which are summarized above have led to the development of many practical protocols. In vitro "chromosome aberration test" in cultured mammalian cells and in vivo "micronucleus assay" in bone marrow cells have been incorporated in the guidelines of mutagenic testing by national and international regulatory bodies. Several international inter-laboratory studies were carried out in 1970 s and 1980 s to standardise the test protocols and these exercises are still going on (Natarajan, 2002).

The main purpose of our research is to determine the damage capacity of mutagens and their capacity for producing different types of chromosomal aberrations and, in perspective, mutations in subsequent generations, the assessment of cytogenetic variability of the modern Ukrainian winter wheat varieties and relationship between cytogenetic parameters and different concentration of DAB.

\section{Material and methods}

Winter wheat seeds (Triticum aestivum L.) (approx. 14\% moisture content) of (in brackets method of obtaining varieties or mutagens used) Favoritka, Lasunya, Hurtovina (irradiation by gamma rays), line 418, Kolos Mironovschiny (field hybridization), Sonechko and Kalinova (chemical mutagenesis), Voloshkova (thermomutagenesis - low plus temperature under vernalization was used as the mutagen factor) were subjected to 1,4-bis-diazoacetyl butane (DAB) $-0,1 \%$ and $0,2 \%$ presoaked. Each treatment used of 1000 wheat seeds. Exposure to chemicals mutagens lasted 18 hours. These concentrations and exposure are optimal for the breeding process, as has been repeatedly established earlier (Ahloowalia et al., 2004; Nazarenko, 2016). Non-treated varieties were used as a control for each variety.

The seeds used in this study were of the $\mathrm{M}_{0}$ generation. After mutagen treatment, the dry seeds were germinated in Petri dishes for 24-48 hours (depending on presoaking and mutagen action), temperature $+25^{\circ} \mathrm{C}$. Afterwards, the central primary roots were cut (if their length was from $10-15 \mathrm{~mm}$ ) and fixed in solution of alcohol and acetic acid (in proportion $3: 1$ ) for 24 hours. The fixation material was stored in $70 \%$ alcohol solution at a temperature of $2{ }^{\circ} \mathrm{C}$ (30-35 roots per variant). We conducted cytological analysis by the standard method by temporary press-time preparations of primary roots tips (1.0-1.5 mm length) stained with acetocarmine (prepared by the Remsdorf method). Tissue maceration was conducted at $45 \%$ solution of acetic acid (during 5 minutes under $60{ }^{\circ} \mathrm{C}$ ). Anaphase of cell division was observed by light microscope JNAVAL. No less than 900 cells in corresponding phases of mitosis were observed in each variant, the number of samples was about 20-25 per variant (Lifang et al., 2001; Rank et al., 2002; Natarajan, 2005).

Statistical analysis of the obtained data was conducted by the method of multi-factor analysis, the variability of the index difference was evaluated by ANOVA, we used the standard tools of the program Statistica 8.0 (StatSoft Inc., USA).

\section{Results and discussion}

In Table 1 we present the data of the results of the following parameters analyzed: total number of observed mitoses in the primary root tips, number of cells in the corresponding phase with visible rearrangements of chromosomal aberrations, total rate of chromosomal aberrations. Standard error $( \pm$ SE) values of the treated variants is shown in Table 1 too. As we can see from Table 1, frequencies of aberrations ranged from $3.3 \%$ (Kalinova, DAB $0.1 \%$ ) to $14.2 \%$ (Voloshkova, DAB $0.2 \%$ ) of the total number of dividing cells in the experimental microscope samples. All the variants are statistically significantly dissimilar from each other and from the control.

Lower frequency of aberrations in any cases was peculiar to varieties obtained by chemical mutation breeding (Sonechko, Kalinova) and we can predict that these varieties are less sensitive to this mutagen (lower rate of visible mutations) (this corresponds to our previous investigations). A higher frequency of aberrations was obtained by use of DAB at $0.2 \%$ as usual.

Rates of chromosomal aberrations were statistically lower when we used DAB for varieties obtained with chemical mutagenesis. The same situation we observed with the varieties Kalinova and Sonechko, when NMU and NEU were used in our previous investi- 
gations, but in this case we cannot see statistically significant difference between these two varieties in contrast to the much sharper decrease for Sonechko under nitrosoalkylureas than for Kalinova.

Table 1

Rate of chromosomal aberrations in winter wheat division cells

\begin{tabular}{|c|c|c|c|c|c|c|}
\hline \multirow{3}{*}{ Variety } & \multirow{2}{*}{$\begin{array}{l}\text { Mitosis, } \\
\text { number }\end{array}$} & \multicolumn{2}{|c|}{$\begin{array}{l}\text { Chromosomal } \\
\text { aberrations }\end{array}$} & \multirow{2}{*}{$\begin{array}{l}\text { Mitosis, } \\
\text { number }\end{array}$} & \multicolumn{2}{|c|}{$\begin{array}{c}\text { Chromosomal } \\
\text { aberrations }\end{array}$} \\
\hline & & number & percent & & number & percent \\
\hline & \multicolumn{3}{|c|}{ Favoritka } & \multicolumn{3}{|c|}{418} \\
\hline Control & 984 & 19 & $1.9 \pm 0.3$ & 962 & 11 & $1.1 \pm$ \\
\hline DA & & 54 & $5.9 \pm 0.7^{*}$ & & 41 & $4.0=$ \\
\hline \multirow[t]{2}{*}{$\mathrm{DAB}, 0.2 \%$} & & 102 & $10.1 \pm 1.0^{*}$ & & 88 & $9.0 \pm 0.9^{*}$ \\
\hline & \multicolumn{3}{|c|}{ Lasunya } & \multicolumn{3}{|c|}{ Hurtovina } \\
\hline Contro & & 15 & $1.4 \pm 0.2$ & & 12 & $1.2 \pm 0.1$ \\
\hline $\mathrm{DAB}, 0$. & & 57 & $5.5 \pm 0.7^{*}$ & & 61 & $6.0 \pm 0.7^{*}$ \\
\hline \multirow[t]{2}{*}{ DAB, $0.2 \%$} & & 104 & $10.2 \pm 1.1^{*}$ & & 111 & $11.2 \pm 1.1^{*}$ \\
\hline & \multicolumn{3}{|c|}{ Sonechko } & \multicolumn{3}{|c|}{ Voloshkova } \\
\hline Contr & 26 & 8 & $0.8 \pm 0.1$ & 1003 & 31 & $3.1 \pm 0.3$ \\
\hline $\mathrm{DAB}, 0$. & & 58 & $5.8 \pm 0,3^{*}$ & 1014 & 81 & $8.0 \pm 0.8^{*}$ \\
\hline \multirow[t]{2}{*}{ DAB, $0.2 \%$} & & 85 & $8.6 \pm 0.5^{*}$ & 979 & 139 & $14.2 \pm 1.1^{*}$ \\
\hline & \multicolumn{3}{|c|}{ Kalinova } & \multicolumn{3}{|c|}{ Kolos Mironivschini } \\
\hline Control & 7 & 9 & $0.9 \pm 0.1$ & 909 & 10 & $1.1 \pm 0.1$ \\
\hline DAB, 0.1 & & 33 & $3.3 \pm 0.1^{*}$ & 1003 & 58 & $5.8 \pm 0.7^{*}$ \\
\hline DAB, $0.2 \%$ & 1013 & 77 & $7.6 \pm 0.4^{*}$ & 1014 & 104 & $10.3 \pm 1.0^{*}$ \\
\hline
\end{tabular}

Note: $*$ - difference statistically significant at $\mathrm{P}<0,05$.

Therefore, we can affirm that DAB as a mutagen by its nature does not so much depend on the object of genotypes as nitrosoalkylureas or less damaging mutagens with lower rates, which are not so evident in their action (by the lower rate of aberrations in similar variants of treatments). The varieties Sonechko and Kalinova are less sensitive to $\mathrm{DAB}$ action. $\mathrm{DAB}$ as a mutagen initiated lower rates of chromosome damage than nitrosoalkylureas or gammarays (Nazarenko, 2016). We can range mutagens in the following sequence according to genetic activity (from the minimum to the maximum) $\mathrm{DAB} \rightarrow \mathrm{NEU} \rightarrow \mathrm{NMU} \rightarrow$ gamma-rays.

From the Table 1 we can see that higher rates of chromosomal changes in any cases are characteristic for varieties obtained by mutation breeding with use of thermo-mutagenesis (Volochkova). All other varieties (field hybridization and after irradiation) were similar in their reaction to $\mathrm{DAB}$ - rate of chromosome rebuilding was around $9-10 \%$. According to this fact, recurrent mutagenesis is an acceptable method for exploiting DAB as a mutagen if we exploit physical mutagens or classical breeding methods for obtaining the objects of action (for example DAB after gamma-rays in our pattern or vice versa).

We found the following types of aberrations of chromosomes after investigation of spectra in our samples: chromosomal bridges and double-bridges, fragments of chromosomes and double-fragments, micronucleus, lagging chromosomes. Cases with complicated aberrations (two or more kinds of changes in one mitosis) and ratio of fragments to bridges were counted separately (Table 2). The number of any type of chromosomal change sharply increased when the concentration was raised (correlation coefficients is $0.6-$ significantly lower than for other mutagens). In this case, as with nitrosoalkylureas, more fragments and double-fragments were caused by DAB (fragments-bridges ratio was more than 1) (Nazarenko and Izhboldin, 2017). We will be able to use this parameter for identifying the difference between the action of gamma-rays and chemical mutagenesis in cases of unknown mutagen factors.

Table 2

Types of chromosomal rebuilding in first generation winter wheat

\begin{tabular}{|c|c|c|c|c|c|c|c|c|c|}
\hline \multirow[t]{2}{*}{ Variant } & \multicolumn{2}{|c|}{$\begin{array}{c}\text { Fragments } \\
\text { (single and double) }\end{array}$} & \multicolumn{2}{|c|}{$\begin{array}{c}\text { Bridges (chromosome } \\
\text { and chromatide) }\end{array}$} & \multirow{2}{*}{$\begin{array}{l}\text { Fragments/ } \\
\text { bridges }\end{array}$} & \multicolumn{2}{|c|}{$\begin{array}{c}\text { Micronucleus, } \\
\text { lagging chromosomes }\end{array}$} & \multicolumn{2}{|c|}{$\begin{array}{l}\text { Complicated } \\
\text { aberrations }\end{array}$} \\
\hline & number & percent & number & percent & & number & percent & number & percent \\
\hline Favoritka, control & 6 & 54.6 & 5 & 45.5 & 1.2 & 0 & 0.0 & 2 & 18.2 \\
\hline $\mathrm{DAB}, 0.1 \%$ & 4 & 21.1 & 14 & 73.7 & 0.3 & 1 & 5.3 & 1 & 5.3 \\
\hline $\mathrm{DAB}, 0.2 \%$ & 41 & 75.9 & 13 & 24.1 & 3.2 & 0 & 0.0 & 0 & 0.0 \\
\hline Lasunya, control & 4 & 26.7 & 11 & 73.3 & 0.4 & 0 & 0.0 & 0 & 0.0 \\
\hline $\mathrm{DAB}, 0.1 \%$ & 37 & 64.9 & 17 & 29.8 & 2.2 & 3 & 5.3 & 0 & 0.0 \\
\hline $\mathrm{DAB}, 0.2 \%$ & 68 & 65.4 & 36 & 34.6 & 1.9 & 0 & 0.0 & 2 & 1.9 \\
\hline Hurtovina, control & 7 & 58.3 & 5 & 41.7 & 1.4 & 0 & 0.0 & 0 & 0.0 \\
\hline $\mathrm{DAB}, 0.1 \%$ & 34 & 55.7 & 27 & 44.3 & 1.3 & 0 & 0.0 & 0 & 0.0 \\
\hline $\mathrm{DAB}, 0.2 \%$ & 59 & 53.2 & 52 & 46.9 & 1.1 & 0 & 0.0 & 4 & 3.6 \\
\hline Line 418 , control & 6 & 54.6 & 5 & 45.5 & 1.2 & 0 & 0.0 & 2 & 18.2 \\
\hline $\mathrm{DAB}, 0.1 \%$ & 29 & 70.7 & 11 & 26.8 & 2.6 & 1 & 2.4 & 0 & 0.0 \\
\hline $\mathrm{DAB}, 0.2 \%$ & 58 & 65.9 & 27 & 30.7 & 2.2 & 3 & 3.4 & 0 & 0.0 \\
\hline Kolos Mironovschiny, control & 5 & 50.0 & 5 & 50.0 & 1.0 & 0 & 0.0 & 0 & 0.0 \\
\hline $\mathrm{DAB}, 0.1 \%$ & 38 & 65.5 & 20 & 34.5 & 1.9 & 0 & 0.0 & 2 & 3.5 \\
\hline $\mathrm{DAB}, 0.2 \%$ & 61 & 58.7 & 37 & 35.6 & 1.7 & 6 & 5.8 & 14 & 13.5 \\
\hline Sonechko, control & 6 & 75.0 & 2 & 25.0 & 3.0 & 0 & 0.0 & 0 & 0.0 \\
\hline $\mathrm{DAB}, 0.1 \%$ & 41 & 70.7 & 17 & 29.3 & 2.4 & 0 & 0.0 & 4 & 6.9 \\
\hline $\mathrm{DAB}, 0.2 \%$ & 63 & 74.1 & 22 & 25.9 & 2.9 & 0 & 0.0 & 8 & 9.4 \\
\hline Kalinova, control & 2 & 22.2 & 7 & 77.8 & 0.3 & 0 & 0.0 & 0 & 0.0 \\
\hline $\mathrm{DAB}, 0.1 \%$ & 18 & 54.6 & 15 & 45.5 & 1.2 & 0 & 0.0 & 0 & 0.0 \\
\hline $\mathrm{DAB}, 0.2 \%$ & 34 & 50.8 & 33 & 49.3 & 1.0 & 0 & 0.0 & 0 & 0.0 \\
\hline Voloshkova, control & 16 & 51.6 & 13 & 41.9 & 1.2 & 2 & 6.5 & 5 & 16.1 \\
\hline $\mathrm{DAB}, 0.1 \%$ & 51 & 63.0 & 30 & 37.0 & 1.7 & 0 & 0.0 & 3 & 3.7 \\
\hline $\mathrm{DAB}, 0.2 \%$ & 86 & 61.9 & 52 & 37.4 & 1.7 & 1 & 0.7 & 7 & 5.0 \\
\hline
\end{tabular}

The number of complicated (or combined) aberrations was significantly lower both for micronuclei and lagging chromosomes than for previous mutagens. Generally, when concentration of DAB was increased the rate of fragments and bridges also increased. But the opposite situation applied to other types. We could not observe these types for the variety Kalinova at all, only fragments and bridges. For line 418, complicated aberrations were also absent, but other types they were present. For varieties Favoritka, Lasunya, Hurtovina (all varieties are radiomutants) in some variants under DAB action complicated aberrations were also absent. On the other hand micronuclei and lagging chromosomes were absent for the varieties Sonechko and Hurtovina. The results of three-factor analysis ("ge- notype", "dose/concentration" and "mutagen") in the general scheme of analysis we include our data from previous investigation of chemical mutagens and gamma-rays action (Nazarenko, 2016), show us that the factors which influence the rate of chromosome aberrations are in order of importance, "dose", then "genotype", then the "mutagen". The role of the second and third factors was increased as compared with our previous investigations. Equally, if we included in the factor scheme only chemical mutagens, we would observe a different situation with the following order of importance, "genotype", "concentration", "mutagen". Thus, we found that repeated exposure to a similar mutagen (for example, $\mathrm{DAB}$ on the variety obtained by the action of this mutagen) leads to 
a substantially lower rate of chromosomal aberrations and absence of some types of rare aberrations in the spectra. Chemical mutagens have been shown to have a higher level of mutagen-genotype interaction and depend more in their action on genotype of the object.

Therefore, investigation of DAB action confirmed the reliability of fragments-bridges ratio (prevalence of fragments over bridges for chemical mutagens and vice versa for gamma-rays) for identification of the nature of a mutagen. Complicated (or combined) aberrations, micronuclei, lagging chromosomes were not observed for some varieties under DAB action. Genotypes selected after action of chemical mutagens are less sensitive to recurrent mutagenesis with chemical mutagens (in case of DAB, NEU, NMU). Previous patterns for other mutagens were confirmed for DAB too.

\section{Conclusions}

$\mathrm{DAB}$ as a mutagen was shown to be substantially lower in chromosomal aberration induction in comparison with previous mutagens (gamma-rays and nitrosoalkylureas). We ranged mutagens in the following sequence (from minimum to maximum) $\mathrm{DAB} \rightarrow \mathrm{NEU} \rightarrow \mathrm{NMU} \rightarrow$ gamma-rays. We can predict a lower amount of mutations if we use DAB for mutation breeding purposes.

The varieties obtained through chemical mutations, Kalinova and Sonechko, are less sensitive to recurrent mutagenesis with this mutagen. In contrast with other chemical mutagens, DAB was less specific in genotype-mutagen interaction on the level of chromosomal rebuilding, but more specific for spectra of aberrations. Complicated (or combined) aberrations were not observed for Kalinova and we did not find micronuclei, lagging chromosomes after DAB action for Kalinova and Sonechko. Chemical mutagens showed a higher level of dependence in its action on the genotype of the object.

Comparison between bridges and fragments following DAB action confirmed reliability of the fragments-bridges ratio (prevalence of fragments over bridges for chemical mutagens and the opposite situation for gamma-rays) for identification of the nature of a mutagen. In general, the rate of chromosomal aberrations showed a linear increase with the concentration of the mutagen.

\section{References}

Adlera, I., Carereb, A., \& Eichenlaub-Ritterc, U. (2004). Gender differences in the induction of chromosomal aberrations and gene mutations in rodent germ cells. Environmental Research, 17, 53-59.

Ahloowalia, B. S., Maluszynski, M., \& Nicoterlein, K. (2004). Global impact of mutation-derived varieties. Euphytica, 135, 187-204.

Ali Sakin, M., Yildirim, A., \& Gikmen, S. (2005). Determining some yield and quality characteristics of mutants induced from a durum wheat (Triticum durum Desf.) cultivar. Turkean Journal Agriculture and Forestry, 29, 61-67.

Albokari, M. (2014). Induction of mutants in durum wheat using gamma irradiation. Pakistan Journal of Botany, 46, 317-324.

Bignold, L. P. (2009). Mechanisms of clastogen-induced chromosomal aberrations: A critical review and description of a model based on failures of tethering of DNA strand ends to strand-breaking enzymes. Mutation Research, 681, 271-298.
Bolzarn, A. D., \& Bianchi, M. S. (2006). Telomeres, interstitial telomeric repeat sequences, and chromosomal aberrations. Mutation Research, $612,189-214$.

Eiges, N. (2013). The historical role of Iosif Abramovch Rapoport in genetics. Further studies using chemical mutagenesis. Russian Journal of Genetics: Applied Research, 3(4), 316-324.

Grant, W. F., \& Owens, E. T. (2001). Chromosome aberration assays in Pisum for the study of environmental mutagens. Mutation Research, 488, 93-118.

Jovtcheva, G., Stergiosa, M., \& Schubert, I. (2002). A comparison of N-methyl$\mathrm{N}$-nitrosourea-induced chromatid aberrations and micronuclei in barley meristems using FISH techniques. Mutation Research, 517, 47-51.

Juchimiuk-Kwasniewska, J., Brodziak, L., \& Maluszynska, J. (2011). FISH in analysis of gamma ray-induced micronuclei formation in barley. Journal of Applied Genetics, 52, 23-29.

Karthika, I. R., \& Subba, B. (2006). Effect of gama rays and EMS on two varieties of soybean. Asian Journal of Biological Sciences, 5, 721-724.

Lifang, W., \& Zengliang, Y. (2001). Radiobiological effects of a low-energy ion beam on wheat. Radiation Environmental Biophysics, 40, 53-57.

Natarajan, A. T. (2002). Chromosome aberrations: Past, present and future. Mutation Research, 504, 3-16.

Natarajan, A. T. (2005). Chromosome aberrations: Plants to human and feulgen to FISH. Current Science, 89, 335-340.

Nazarenko, M. (2017). Specific features in the negative consequences of a mutagenic action. Russian Journal of Genetics: Applied Research, 7(2), 195-196.

Nazarenko, M. (2016). Parameters of winter wheat growing and development after mutagen action. Bulletin of Transilvania University of Brasov - series II - Forestry, Wood Industry, Agricultural, Food Engineering, 58(2), 109-116

Nazarenko, M. (2016). Specify of nitrosoalkylureas action on cell level in winter wheat. Visnyk of Dnipropetrovsk University. Biology, Ecology. 24(2), 258-263.

Nazarenko, M., \& Izhboldin, O. (2017). Chromosomal rearrangements caused by gamma-irradiation in winter wheat cells. Biosystems Diversity, 25(1), 25-28.

Nikolova, I., Georgieva, M., Kruppa, K., Molnor-Long, M., Liu, L., Manova, V., \& Stoilov, L. (2015). Cytogenetic effects in barley root apical meristem after exposure of dry seeds to lithium ion beams. Genetics and Plant Physiology, 5, 3-9.

Rakhmatullina, E. M., \& Sanamyan, M. F. (2007). Estimation of efficiency of seed irradiation by thermal neutrons for inducing chromosomal aberration in $\mathrm{M}_{2}$ of cotton Gossypium hirsutum L. Russian Journal of Genetics, 43(5), 518-524.

Rank, J., Lopez, L. C., \& Nielsen, M. H. (2002). Genotoxicity of maleic hydrazide, acridine and DEHP in Allium cepa root cells performed by two different laboratories. Hereditas, 136, 13-18.

Shu, Q. Y., Forster B. P., \& Nakagava, H. (2011) Plant mutation breeding and biotechnology. CABI publishing, Vienna.

Ukai, Y. (2006). Effectiveness and efficiency of mutagenic treatments. Gamma Field Symposia, 45, $1-4$.

de Vries, H. (1918). Mass mutations and twin hybrids in Oenothera grandiflora Ait. Botanical Gazette, 65, 377-422.

Waugha, R. (2006). Harvesting the potential of induced biological diversity. Trends in Plant Science, 11, 71-79.

Zhang, J., Jiang, Y., Guo, Y., Li, G., Yang, Z., Xu, D., \& Xuan, P. (2015). Identification of novel chromosomal aberrations induced by ${ }^{60} \mathrm{Co}-\gamma-$ irradiation in wheat - Dasypyrum villosum lines. Intternational Journal of Molecular Sciences, 16, 29787-29796. 